\title{
Acute Angioedema in a Patient on Long-term Angiotensin Converting Enzyme Inhibitor Following Oral Surgery: A Case Report
}

\author{
Caleb A. Awoniyi*,1,2, Steven Yannaras ${ }^{2}$ and Julia M. Bauerfeind ${ }^{1,2}$ \\ ${ }^{I}$ North Florida/South Georgia Veterans Health System, Anesthesia Service, Gainesville, Florida, USA \\ ${ }^{2}$ Department of Anesthesiology, University of Florida, Gainesville, Florida, USA
}

\begin{abstract}
A relatively common side effect of angiotensin converting enzyme inhibitors (ACE-I), used for the treatment of high blood pressure, is angioedema. Whereas, angioedema due to ACE-I usually appear during the first few days or weeks of treatment, delayed onset, years after initiation of treatment is often unrecognized and can lead to life-threatening adverse events. We present a case report of rapidly progressive tongue swelling a few hours following oral surgery in a 62-year-old male who had been on ACE-I therapy for seven years. Prompt recognition and treatment prevented an impending respiratory compromise that could have been catastrophic.
\end{abstract}

Keywords: Angiotensin converting enzyme inhibitor, angioedema, late-onset of angioedema, tongue swelling, hereditary angioedema, nasal intubation.

\section{INTRODUCTION}

Angioedema is a well-recognized side effect of angiotensin converting enzyme inhibitor (ACE-I) therapy [1]. In fact, ACE-I are the leading cause of drug -induced angioedema in the United States accounting for up to $40 \%$ of all emergency room visits for angioedema each year. Commonly, patient presents with swelling of the lips, tongue, or face, although, intestinal angioedema has also been described. This ACE-I induced angioedema is idiosyncratic and unpredictable. It can occur early in treatment with about $60 \%$ of cases occurring within the first few days [2], or after a prolonged therapy of several years $[3,4]$. In addition, some cases of ACE-I induced angioedema have been reported to occur following discontinuation of the drug.

\section{CASE REPORT}

A 62 year-old male with a supraglottic lesion presented for panendoscopy. His past medical history was significant for tobacco use disorder, alcohol dependence, hypertension, gastroesophageal reflux disease, and osteoarthritis. His usual medications included etodolac, hydrochlorothiazide, lisinopril, metoprolol, and omeprazole. He was allergic to amlodipine. Of note, the patient had been on lisinopril for seven years. Prior to his surgery, he had experienced hoarseness for about 3 months and was evaluated by ENT. He was noted to have a supraglottic mass that was centered at the midline of the infrahyoid epiglottis with extension to the anterior false vocal cords. There was no apparent adenopathy or metastatic disease. His pre-operative

*Address correspondence to this author at the North Florida/South Georgia Veterans Health System, Anesthesia Service, Gainesville, Florida, USA; Tel: 352-374-6051; Fax: 352-379-7489; Email: Caleb.awoniyi@va.gov evaluation included a blood pressure of $146 / 81 \mathrm{~mm} \mathrm{Hg}$, heart rate of 75 , respiratory rate of 18 breaths per minute, and pulse oximetry oxygen saturation of $98 \%$. His EKG and laboratory tests were unremarkable. The patient's airway was patent with Mallampati III classification. The cervical spine had free range of motion. On the day of surgery, the patient was taken to the operating room, routine induction of anesthesia took place, a Glidescope was used to visualize the vocal cords and a 6.5 endotracheal tube was easily passed. Throughout the surgical course, that lasted about one hour, the patient remained hemodynamically stable. He was extubated without incidence and was taken to the postanesthesia care unit. After an uneventful recovery in Phase 1 of post anesthesia recovery unit (PACU) for approximately an hour until anAldrete score of 12 could be documented, he was taken to Phase II PACU with the plan to be discharged home. While in Phase II recovery, his tongue was noted to be swelling. Initial evaluation showed a moderately edematous tongue, the oral airway was patent and the patient denied any respiratory distress. He was given intravenous dexamethasone $(8 \mathrm{mg})$, ranitidine $(50 \mathrm{mg})$, and diphenhydramine $(25 \mathrm{mg})$. However, over the course of about 20 minutes, his tongue swelling and floor of mouth edema worsened. In addition, his upper and lower lips had also become swollen (Fig. 1A). Due to the progressive worsening of his tongue, lip and airway edema despite medical therapy, he was taken to the operating room for elective awake fiberoptic nasal intubation (Fig. 1B); the ENT surgeon had been notified and was present in the operating room during intubation in case a surgical airway would become necessary. The patient was successfully nasally intubated, sedated and taken to the intensive care unit. His lisinopril was discontinued. His tongue, lip, and airway 


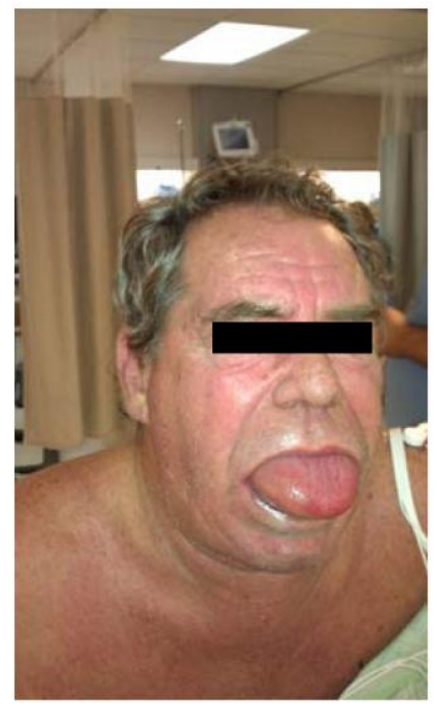

A

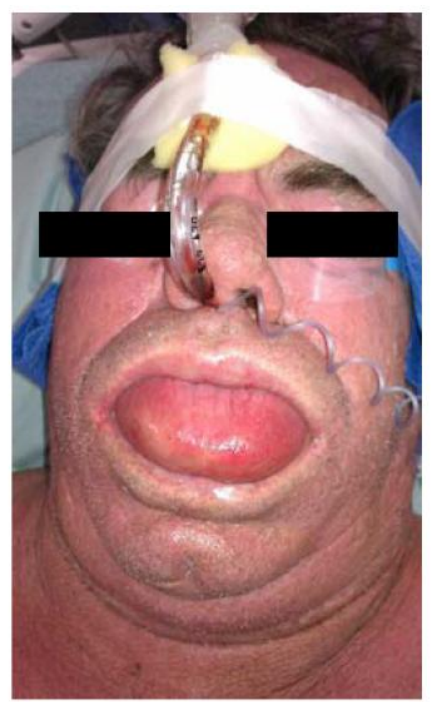

B
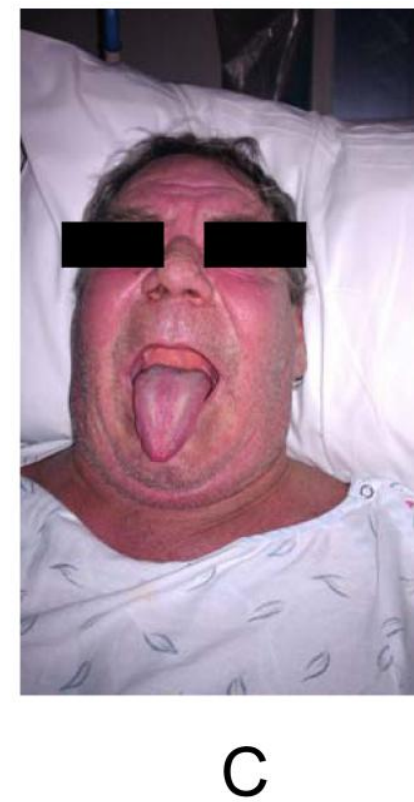

Fig. (1). A) enlarged tongue prior to going to the OR; B) patient's airway secured with nasal intubation, and C) patient extubated after resolution of symptoms

edema resolved over 48 hours, and he was extubated (Fig. 1C) on postoperative day 2 .

\section{DISCUSSION}

Angioedema involving the tongue and throat can be fatal due to airway obstruction and asphyxia. The differential diagnosis of post-operative tongue and lip swelling includes allergic reactions, anaphylaxis, venous congestion of the tongue (secondary to prolonged compression during surgery), hereditary angioedema (HAE) or acquired angioedema. Our patient's clinical evaluation appeared to be consistent with acquired angioedema, likely drug induced. He was hemodynamically stable without systemic or dermatological manifestations that would suggest anaphylaxis or allergic reaction. His list of medications was reviewed and showed no indication of any inadvertent drug administration. Although HAE is unlikely in this 62 year old patient, given the lack of HAE family history, it is an important factor to consider in this scenario. HAE is a relatively rare autosomal dominant condition caused by a deficiency in C-1 inhibitor and differs from acquired angioedema, such as drug-induced. The onset of symptoms for HAE typically occurs in childhood, an increased incidence seen during puberty and recurrent attacks occurring throughout life [5]. In addition, HAE attacks are often spontaneous without any identifiable triggers [6]. Drug induced angioedema, however, differs from HAE by the absence of a family history, onset of symptoms later in life and commonly, the presence of a trigger. For acute attacks of HAE, treatment modalities, such as epinephrine, corticosteroids or antihistamine do not have salutatory effect and are not recommended [7]. Although there is insufficient evidence to support administration of epinephrine, antihistamines and corticosteroids in the treatment of acquired angioedema [8], most agree that once diagnosis of ACE-I induced angioedema is made, the drug should be stopped and the patient should receive oxygen and other supportive measures [9]. Knowing that there have been no reports of adverse events after giving symptomatic support with epinephrine, antihistamines and corticosteroids, it is likely that these drugs will continue to be given in this situation until the symptoms subside with natural progression after 24-48 hours.

The etiology of this patient's angioedema is likely the result of the use of lisinopril, and/or surgery involving his oropharynx. In most cases of drug-induced angioedema of the tongue, ACE-I have always been the major offenders, occurring $25-58 \%$ of the time [10]. With ACE-I induced angioedema, the reaction usually occurs during the first few weeks of treatment. However, there have been isolated cases of delayed angioedema in patients on long-standing ACE inhibitor therapy, as probably is the case in our example. The prevalence of delayed onset angioedema may increase, given the growing numbers of patients on ACE-I. Other less common causes of drug-induced angioedema include angiotensin-2 receptor antagonists, statins, NSAIDs, bupropion [11], metoprolol, SSRIs, calcium channel blockers, amiodorone, and risperidone [12-15], none of which the patient was taking at the time.

Maxillofacial and head and neck surgery have also been implicated in the development of oral angioedema in patients taking ACE-I [16-18]. Therefore, a delayed hypersensitivity reaction to ACE inhibitors exacerbated by panendoscopic surgery may have accounted for this patient's symptoms. It is possible that the surgical trauma of the oral mucosa and submucosa initiated a surge in inflammatory mediators that triggered such a response.

Although the etiology of the development of ACE-I induced angioedema is still not known, bradykinin-induced activation of vascular bradykinin beta- 2 receptors has been proposed. ACE is the most important enzyme regulating the breakdown of bradykinin in plasma and tissue leading to 
induction of vasodilation and vascular leakage, mainly by activation of endothelial bradykinin-2 receptor $[19,20]$, suggesting that ACE-I substantially increase the steady-state level of bioactive bradykinin. Some studies have shown increased levels of bradykinin in patients with ACE-I induced angioedema versus controls; however, patients with ACE dysfunction do not have increased rates of angioedema [21]. Trauma can increase concentrations of vasoactive substances and may be responsible for the connection between oral surgical manipulation and angioedema. Thus, therapeutic effect of Icatibant, a bradykinin beta- 2 receptor inhibitor has shown some promise to ameliorate this problem, possibly even to completely relieve angioedema altogether [22]. The bradykinin-mediated theory is further supported by the fact that most ACE-I induced angioedema is unresponsive to standard care with corticosteroids, antihistamine, or epinephrine. The mainstay of treatment is supportive, directed towards airway protection.

In summary, the fact that a patient has been on long-term ACE-I therapy does not negate the development of angioedema. Because ACE-I are now widely prescribed for the treatment of hypertension, as well as to provide cardiovascular and renal protection in patients at risk for cardiovascular events, heart failure, and kidney disease, the attributable risk of angioedema due to ACE-I will also increase. Messerli et al, suggest that considering that several million patients worldwide are treated with ACE-I, this drug class could account for several hundred deaths per year due to laryngeal edema [23]. Had this patient's delayed angioedema developed after being discharged, there is possibility that a fatal outcome could have occurred. Currently, there is not enough data regarding ACE-I and oral surgery induced angioedema to infer that combinations of both could induce angioedema. However, there is a trend in the literature of case studies that suggest that the combination may play a role. Perhaps, patients on ACE-I having oral surgeries should be observed longer in the PACU before being discharged home. By and large, there should be increased vigilance and prompt recognition of impending catastrophic airway collapse in this group of patients so that appropriate treatment, such as elective nasal intubation, is provided promptly.

\section{CONFLICT OF INTEREST}

The authors confirm that this article content has no conflicts of interest.

\section{ACKNOWLEDGMENTS}

None declared.

\section{REFERENCES}

[1] Agah R, Bandi V, Guntupalli KK. Angioedema: the role of ACE inhibitors and factors associated with poor clinical outcome. Intensive Care Med 1997; 17: 793-96.

[2] Hedner T, Sameulsson O, Lunde H, Lindholm L, Wiholm BE. Angio-edema in relation to treatment with angiotensin concerting enzyme inhibitors. BJM 1992; 304: 941-6.

[3] Sondhi D. Lippmann M, Murali G. Airway compromise due to angiotensin-converting enzyme inhibitor-induced angioedema: clinical experience at a large community teaching hospital. Chest 2004; 126: 400-4.

[4] Dyer PD. Late-onset angioedema after interruption of angiotensinconverting enzyme inhibitor therapy. J Allergy Clin Immunol 1994; 93: 947-48.

[5] Cicardi M, Johnson DT. Hereditary and acquired complement component I esterase inhibitor deficiency: a review for the hematologist. Acta Hematol 2012; 127: 208-20.

[6] Gompels MM, Lock RJ, Abinun M, et al. CI inhibitor deficiency: consensus document. Clin Exp Immunol 2005: 139: 379-94.

[7] Bowen $\mathrm{T}$, Cicardi M, Farkas $\mathrm{H}$, et al. International consensus algorithm for the diagnosis, therapy and management of hereditary angioedema. Allergy Asthma Clin Immunol 2010; 6: 24

[8] Winter ME, Rosenbaum S, Vilke GM, Almazroua FY. Emergency department management of patients with ACE-inhibitor angioedema. J Emerg Med 2013, (in press).

[9] Zirkle M, Bhattacharyya N, Predictors of airway intervention in angioedema of the head and neck. Otolaryngol Head Neck Surg 2000; 123: 240-5.

[10] Cohen EG, Soliman AM. Changing trends in angioedema. Ann Oto Rhin Larngol 2001; 110: 701-6.

[11] Sanchez-Borges M, Gonzalez-Aveledo LA. Angiotensin converting enzyme inhibitors and angioedema. Allergy Asthma Immunol Res 2010; 2: 195-8.

[12] Mithani, H, Hurwitz TA. Paroxetine-induced angioedema and tongue swelling [letter]. J Clin Psychiatry 1996; 57: 486.

[13] Cooney C, Nagy A. Angio-oedema associated with risperidone. BJM 1995; 311: 1204.

[14] Burches E, Garcia-Verdegay F, Ferrer M, Pelaez A. Amiodaroneinduced angioedema. Allergy 2000; 55: 1199-200.

[15] Krikorian RK, Quick A, Tal A, et al. Angioedema following the intravenous administration of metoprolol. Chest 1994; 106: 192223.

[16] Reed LK, Meng J, Joshi GP. Tongue swelling in the recovery room: a case report and discussion of postoperative angioedema. J Clin Anesth 2006; 18: 226-9.

[17] O'Cannor R, Lau C, Cameron M, Thompson M. ACE inhibitors and angioedema in oral and maxillofacial surgery: Thoughts for the trainee. Face Mouth Jaw Surg 2011; 1:22-25.

[18] Ogbureke KUE, Cruz C, Johnson JVE et al. Perioperative angioedema in a patient on long-term angiotensin-converting enzyme (ACE)-inhibitor therapy. J Oral Maxillofac Surg 1996; 54: 917-20, 1996

[19] Han ED, MacFarlane RC, Mulligan AN, et al. Increased vascular permeability in $\mathrm{C} 1$ inhibitor-deficient mice mediated by the bradykinin type 2 receptor. J Clin Invest 2002; 109: 1057-63.

[20] Davis AE. Biological effects of C1 inhibitor. Drug News Perspect 2004; 17: 439-46.

[21] Vleeming W, van Amsterdam JG, Stricker BH, de Wildt DJ. ACE inhibitor-induced angioedema.Incidence, prevention and management. Drug Saf 1998; 18: 171-88.

[22] Bas M, Greve J, Stelter K, et al. Therapeutic efficacy of ictibant in angioedema induced by angiotensin-converting enzyme inhibitors: A case series. Ann Emerg Med 2010; 56: 278-82.

[23] Messerli FH, Nussenberger J. Vasopeptidase inhibition and angioedema. Lancet 2000; 356: 608-9. 